\title{
CONHECIMENTO ETNOMICOLÓgICO DE COMUNIDADES QUE HABITAM O ENTORNO da REBiO de Pedra Talhada, Alagoas, Brasil
}

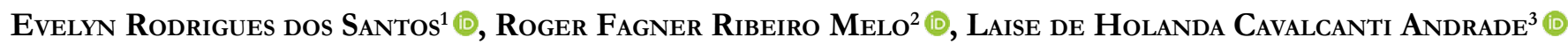

${ }^{1}$ Discente do Programa de Pós-Graduação em Biologia de Fungos, Universidade Federal de Pernambuco. Av. da Engenharia, s/n, Cidade Universitária. CEP. 50740-570.

${ }^{2}$ Docente do Departamento de Micologia, Centro de Biociências, Universidade Federal de Pernambuco. Av. Prof. Moraes Rego, s/n ${ }^{\circ}$, Cidade Universitária. CEP: $50.760-420$.

${ }^{3}$ Docente do Departamento de Botânica, Centro de Biociências, Universidade Federal de Pernambuco. Av. Professor Moraes Rego, S/N - Cidade Universitária, Recife - PE. CEP: 50670-420.

* Autorpara correspondência: evlinha@hotmail.com

Recebido em 07 de fevereiro de 2020. Aceito em 06 de julho de 2020. Publicado em 31 de julho de 2020.

Resumo - Estudos etnomicológicos sobre comunidades brasileiras são escassos, quase todos centrados em povos da Amazônia. O presente trabalho teve por objetivo verificar a existência de conhecimento etnomicológico em nove comunidades nordestinas estabelecidas nas proximidades da Reserva Biológica de Pedra Talhada - RBPT (Quebrangulo, AL). Utilizou-se um formulário semiestruturado, um álbum de fotografias e amostras de macrofungos recém-coletadas para entrevistar 61 pessoas (59\% homens; 41\% mulheres; 18 a 81 anos), a maioria agricultores (70\%) com baixo nível de instrução. Quase todos $(92 \%)$ reconheceram os macrofungos e a maioria citou seus nomes populares, baseados em caracteres morfológicos, associados ao ambiente onde ocorrem (cebola da mata, cogumelo do cocô, orelha de pau) ou às propriedades a eles atribuídas (cogumelo do bem e cogumelo do mal). Percebem a relação desses organismos com o ambiente e a influência dos fatores climáticos sobre sua esporulação. Chás preparados com basidiomicetos foram indicados para tratamento de câncer (Fomes fasciatus) e asma (Ganoderma australe); duas espécies não identificadas foram indicadas para tratamento de problemas respiratórios. Cookeina tricholoma (Ascomycota) foi referida como comestível por um ex-caçador idoso. O uso lúdico foi relatado para uma espécie de Geastrum (Basidiomycota). Embora não micófilos, os entrevistados percebem os macrofungos como elementos importantes no ambiente.

Palavras-chave: Etnobiologia; Etnomicologia; Macrofungos; Mata Atlântica; Micologia.

Conocimiento etnomicológico de comunidades que habitan en el entorno de la Reserva Biológica de Pedra Talhada, Alagoas, Brasil

Resumen - Son escasos los estudios etnomicológicos de comunidades brasileñas, y casi todos los estudios están centrados en pueblos de la Amazonía. El presente trabajo tuvo como objetivo verificar la existencia de conocimiento etnomicológico en nueve comunidades nordestinas establecidas en la vecindad de la Reserva Biológica de Pedra Talhada - RBPT (Quebrangulo, AL). Se utilizó un formulario semiestructurado, un álbum fotográfico y muestras de macrohongos recién colectados para entrevistar a 61 personas (59\% hombres; 41\% mujeres, de 18 a 81 años), la mayoría agricultores (70\%) con bajo nivel de educación. Casi todos (92\%) reconocieron a los macrohongos, y la mayoría dijo sus nombres vulgares basándose en características morfológicas asociadas al ambiente en donde ocurren (cebolla del bosque, seta de la caca, oreja de judas) o a las propiedades a ellas asignadas (seta del bien y seta del mal). Reconocen la relación entre estos organismos y la influencia de los factores climáticos sobre su esporulación. Se indicaron infusiones preparadas con basidiomicetos para el tratamiento de cáncer (Fomes fasciatus) y asma (Ganoderma australe); dos especies no identificadas fueron indicadas para el tratamiento de problemas respiratorios. La Cookeina tricholoma (Ascomycota) fue referida como comestible por un señor mayor ex-cazador. También fue relatado el uso lúdico de una especie de Geastrum (Basidiomycota). A pesar de no micófilos, los entrevistados reconocen a los macrohongos como elementos importantes en el medio ambiente. 
Palabras clave: Etnobiología; Etnomicología; Macrohongos; Bosque Atlántico; Micología.

Ethnomycological knowledge of communities that inhabit the sorroundings of Pedra Tahada Biological Reserve, Alagoas, Brasil

ABstract - Ethnomycological studies on Brazilian communities are scarce, almost all of it focused on Amazonian natives. The present study aimed to access the extent of ethnomycological knowledge in nine northeastern communities established in the vicinity of the Pedra Talhada Biological Reserve - RBTP (Quebrangulo, Alagoas State, Brazil). A semi-structured form, a photo album and fresh samples of macrofungi were used in the interviews of 61 individuals (59\% men; 41\% women, aged from 18 to 81 years old), most of them subsistence farmers (70\%) with low level of instruction. Almost all of them (97\%) recognized the macrofungi and cited their popular names, based on morphological characters, associated with the environment where they occur (onion-of-the-forest, dung-mushroom, wood-ear) or the properties attributed to it (good and evil mushrooms). They perceive the relationship of these organisms with the environment and the influence of climatic factors on their sporulation. Teas prepared with basidiomycetes were indicated for cancer treatment (Fomes fasciatus) and asthma (Ganoderma australe); two unidentified species were indicated for the treatment of respiratory problems. Cookeina tricholoma (Ascomycota) was referred as edible by an elderly former hunter. Ludic use has been reported for a species of Geastrum (Basidiomycota). Although not considered mycophiles, the interviewees perceive macrofungi as important elements in their environment.

KEYwORDs: Ethnobiology; Ethnomycology; Macrofungi; Atlantic forest; Mycology.

\section{INTRODUÇÃO}

Desde as primeiras civilizações o homem se relaciona com os fungos, como evidenciam os achados efetuados no Alpes Suíços, quando foi encontrado o chamado Ötzi, o homem do gelo, que viveu no Neolítico e portava pelo menos três espécies de fungos, Fomes fomentarius (L.) Fr., Fomitopsis betulina (Bull.) B.K. Cui, M.L. Han \& Y.C. Dai e Inonotus obliquus (Ach. ex Pers.) Pilát, que provavelmente utilizava como medicinais e alimentícios (Kotowski 2019). Os primeiros estudos etnomicológicos já evidenciavam que, enquanto algumas sociedades utilizam largamente os cogumelos na alimentação, além de outros usos, como medicinal e mágico-ritualístico, outras desenvolveram certa aversão a eles, tratando-os como venenosos ou, no mínimo, indigestos. Wasson (1957) denominou essas relações como micofílicas, as primeiras e como micofóbicas as últimas.

Em um mesmo continente, algumas culturas podem ser consideradas micofílicas enquanto outras são caracteristicamente micofóbicas. Na Europa, por exemplo, povos da região do Mediterrâneo são reconhecidamente micófilos enquanto os da região do Atlântico são micófobos (Mapes et al. 2002). Os russos se destacam pela forte apreciação dos fungos como comestíveis, enquanto os alemães, de modo geral, são micófobos e normalmente não usam fungos como alimento, pois lhes despertam fortes emoções negativas, e evitam tocar nos esporocarpos, que consideram como sujos ou causadores de doenças (Mapes et al. 2002).

Documentos arqueológicos mostram a forte relação povo x fungo, particularmente sob o aspecto místicoritualista, em países latino-americanos, como México, Peru e Guatemala (Morales et al. 2003; Ruan-Soto 2017; Trutmann et al. 2012). Em artigo sobre uso de recurso micológico pelos Patamona, comunidade tradicional da Guiana, Henkel et al. (2004) comentam que muitas das espécies comestíveis para o povo local pertencem a famílias não apreciadas como alimento por norte-americanos, como Clavulinaceae, Sarcoscyphaceae e Amanitaceae, enquanto grupos altamente apreciados em regiões de clima temperado, como Cantharellaceae, Boletaceae e Polyporaceae, são por eles evitados. Povos da Amazônia brasileira, onde existe uma rica micobiota, possuem terminologia bem desenvolvida para se referir aos fungos, mas poucos grupos são reconhecidos como micófilos (Góes-Neto e Bandeira 2002; Santana et al. 2019). 
Estudos sobre as relações povo $\mathrm{x}$ fungos no Brasil são escassos e esparsos ao longo do tempo, quase todos centrados na Região Norte, com foco nos macrofungos da Amazônia (Prance 1973; Fidalgo e Prance 1976; Fidalgo e Poroca 1986; Góes-Neto e Bandeira 2002; Cardoso et al. 2010; Ishikawa et al. 2012; Vargas-Isla et al. 2013; Apiamö et al. 2016; Yanomami et al. 2019), sendo raros e recentes os desenvolvidos nas Regiões Nordeste, com comunidades rurais e indígenas da Caatinga (Sousa et al. 2015, 2017a, 2017b; Londoño-Castañeda et al. 2017; Sousa 2020), Centro-Oeste (Cunha 2019) e Sul (Trierveiler-Pereira et al. 2018).

As restrições legais referentes a áreas oficialmente protegidas, embora preservem a biodiversidade, podem influenciar negativamente a geração e transmissão do etnoconhecimento (Zuchiwschi et al. 2010), especialmente as de preservação integral, como as Reservas Biológicas. Até a década de 1980, muitas famílias tinham casas, pequenas lavouras e animais domésticos dentro dos fragmentos florestais que compõem a REBIO de Pedra Talhada, situada nos limites dos estados de Pernambuco e Alagoas, no Nordeste do Brasil. É possível que a diminuição do contato direto e diário com os elementos da biota local nas três últimas décadas, após ter sido criada a Unidade de Conservação, tenha interferido na geração e transmissão do etnoconhecimento existente em populações que vivem no entorno da REBIO de Pedra Talhada.

No presente trabalho, o primeiro a ser realizado sobre as relações de comunidades com macrofungos da Floresta Atlântica nordestina, investigou-se o conhecimento etnomicológico de homens e mulheres de diferentes faixas etárias, moradores no entorno da REBIO de Pedra Talhada, visando defini-los como micófilos ou micófobos.

\section{Material e MÉtodos}

Área de estudo

A Reserva Biológica de Pedra Talhada - RBPT, criada em 1989, abrange 4.469 ha, distribuídos em dois municípios de Alagoas (Quebrangulo e Chã Preta) e dois de Pernambuco (Lagoa do Ouro e Correntes), entre as coordenadas $36^{\circ} 22^{\prime}$ a $36^{\circ} 28^{\prime} \mathrm{O}$ e $9^{\circ} 11^{\prime}$ a $9^{\circ} 16^{\prime} \mathrm{S}$. O clima de Pedra Talhada é do tipo tropical, marcado por uma estação seca, de setembro a fevereiro, com pluviosidade mensal com menos de $50 \mathrm{~mm}$, e uma estação chuvosa, de março a agosto, com pluviosidade mensal de $250 \mathrm{~mm}$. A temperatura média anual varia entre $23^{\circ} \mathrm{C}$ e $25^{\circ} \mathrm{C}$, e a umidade atmosférica entre 35\% e 90\%, devido à presença da Serra das Guaribas, permitindo a formação de um brejo de altitude, ou seja, uma ilha de floresta úmida circundada pela vegetação típica da região semiárida nordestina (Nusbaumer et al. 2015).

A pesquisa foi realizada em comunidades rurais estabelecidas no município de Quebrangulo, que corresponde a cerca de 60\% da área da reserva, com residências distribuídas nas proximidades da RBPT (Figuras 1 e 2). De acordo com o IBGE (2010), a população desse município, de característica rural, compreende 11.480 habitantes (densidade demográfica de $36 \mathrm{hab} / \mathrm{km}^{2}$ ), distribuída em um território de $320 \mathrm{~km}^{2}$, a uma altitude média de 350 m (Tscharner et al. 2015). 
Figura 1. Mapa de localização das comunidades do entorno da REBIO de Pedra Talhada (Juliana, Carangueja, Pedra Talhada, Sítio do Gavião, 5 Riachão de Baixo e Riachão de Cima, Pai Vicente, Arraial e Boa Vista), município de Quebrangulo, Alagoas, Brasil.
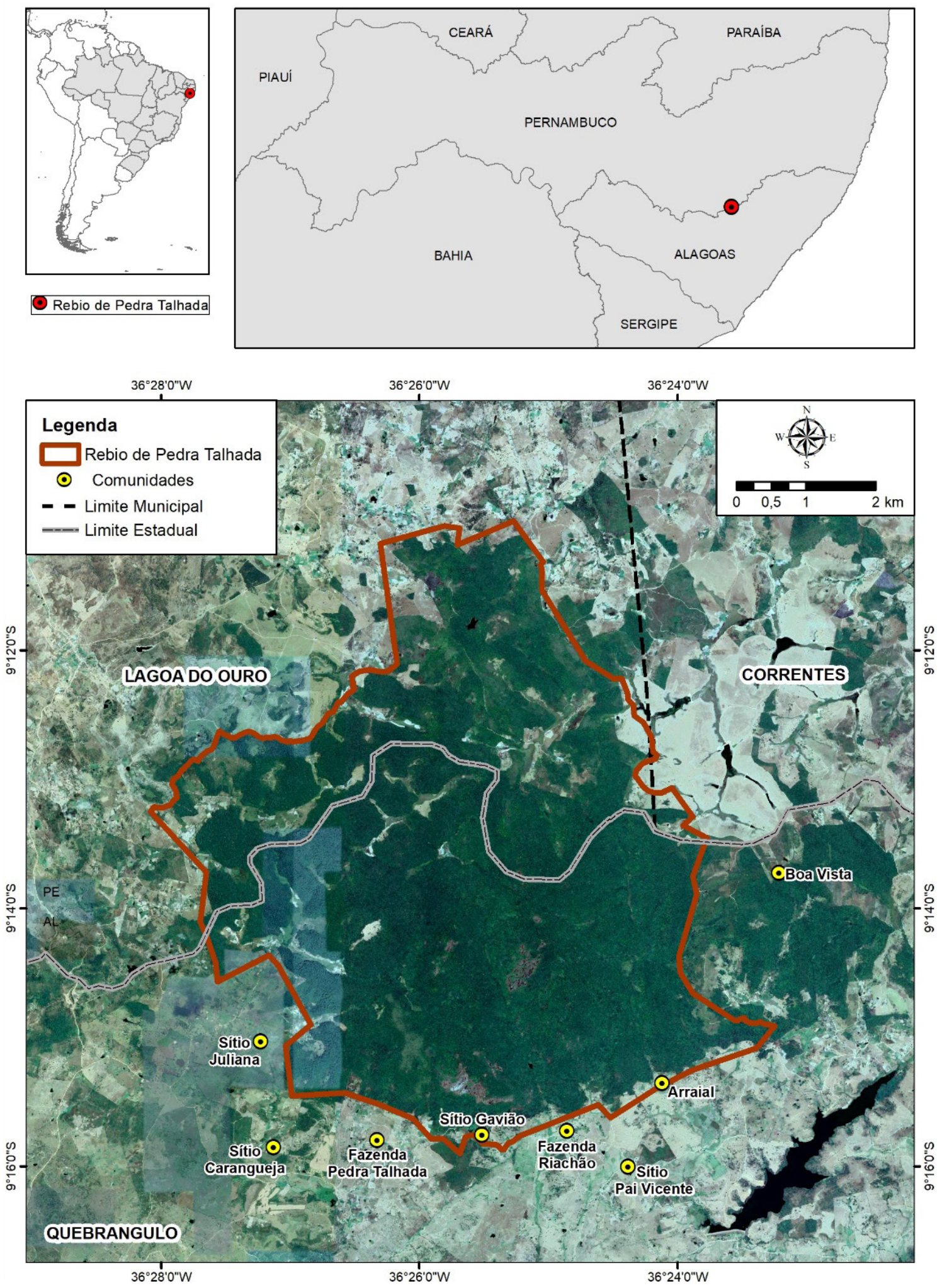

Fonte: Os autores. 
Figura 2. A) Casa de alvenaria e telhas de barro, tipo de moradia mais comum encontrada nas comunidades que moram no entorno da Reserva Biológica de Pedra Talhada - RBT, Quebrangulo, Alagoas. B) Comunidade do Riachão de Cima: moradias distanciadas, distribuídas ao longo da estrada de barro; ao fundo a floresta da RBPT.

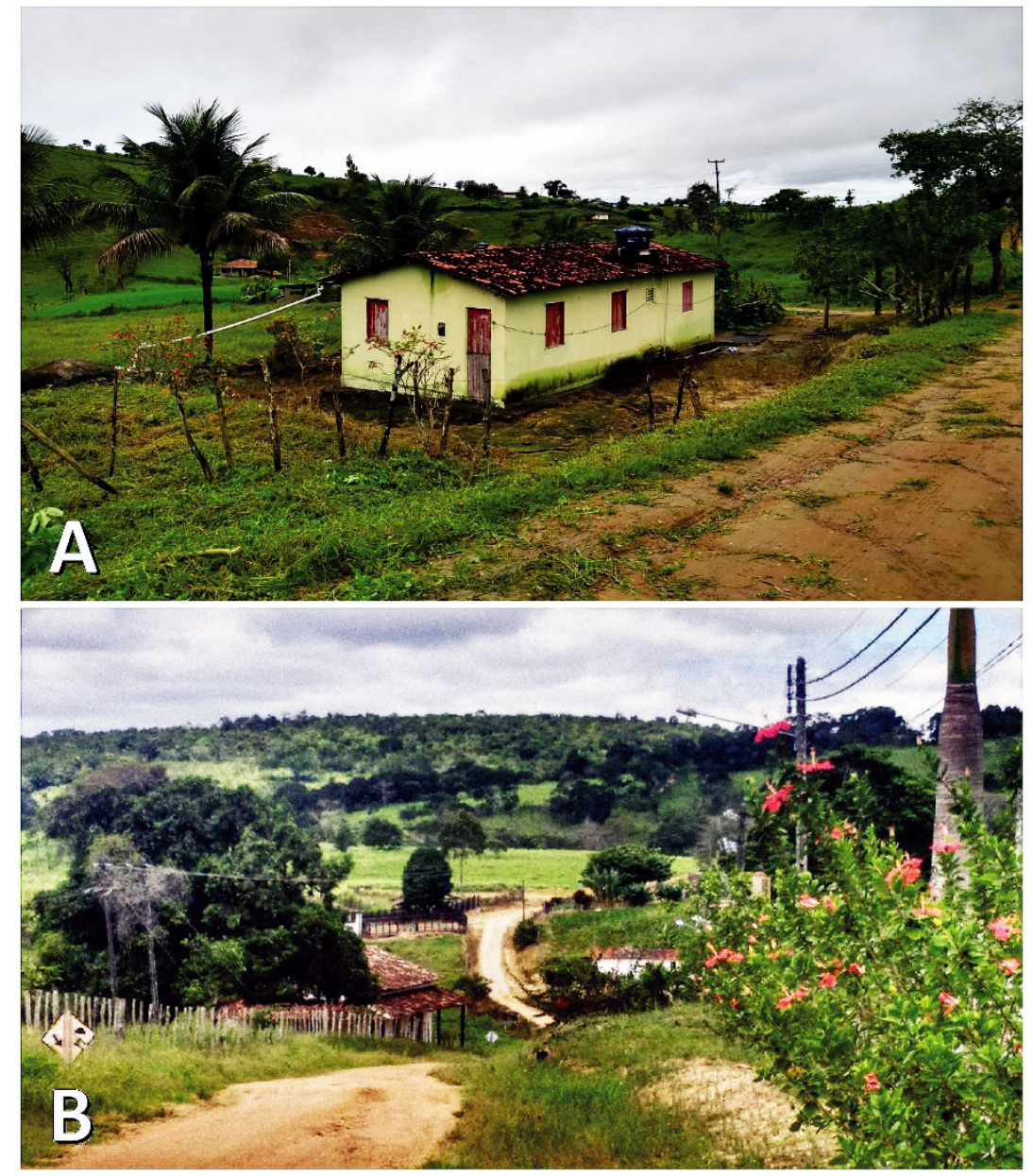

\section{Coleta de informações etnomicológicas}

Uma excursão piloto foi realizada em julho de 2018, para obtenção de carta de anuência do gestor da Associação Comunitária e primeiros contatos com moradores das comunidades. Nos meses de fevereiro, maio e agosto de 2019 foram realizadas excursões, com duração de sete dias cada, para coleta de macrofungos, contato com os moradores e entrevistas.

Os dados etnomicológicos foram coletados a partir de entrevistas com moradores das comunidades de Pedra Talhada, Carangueja, Sítio do Gavião, Juliana, Arraial, Riachão de Cima, Riachão de Baixo, Pai Vicente e Boa Vista (Figura 1), homens e mulheres, a partir da idade de 18 anos, selecionados através da técnica conhecida como bola de neve (Albuquerque et al. 2008). Como critério de divisão dos grupos por faixa etária, seguiu-se a delimitação utilizada pelo Instituto Brasileiro de Geografia e Estatística (IBGE 2010): jovens (entre 18 e 24 anos), adultos (entre 25 e 59 anos) e idosos ( $\leq 60$ anos).

Aplicou-se um formulário semiestruturado com 61 informantes ( 36 homens e 25 mulheres) após autorização do participante (Termo de Consentimento Livre e Esclarecido), atendendo aos requisitos do Comitê de Ética em Pesquisa da Universidade Federal de Pernambuco - UFPE (CAAE: 96880818.8.0000.5208), e à Resolução 466/2012 da Comissão Nacional de Ética e Pesquisa do Ministério da Saúde. Além dos dados sócio-econômicos dos entrevistados (faixa etária, ocupação principal, tempo de moradia, faixa de renda e nível de instrução), foram formuladas questões abertas sobre como reconhecem e identificam os fungos, época do ano e ambientes onde 
são encontrados e se fazem algum tipo de uso das espécies que ocorrem próximas às suas residências e lavouras e no interior da RBPT.

Como recursos complementares, foram utilizados estímulos visuais (Figura 3), como uma estratégia para induzir as pessoas a lembrarem de determinadas informações que interessavam à pesquisa. Apresentou-se a cada entrevistado um álbum de fotografias de macrofungos que ocorrem na RBPT e uma caixa com amostras de esporocarpos recém-coletadas em campo, contendo espécies de Amauroderma, Cantharellus, Chlorophyllum, Cladonia, Cookeina, Fomes, Ganoderma, Geastrum, Herpothallon, Macrolepiota, Pycnosporus, Schizophyllum e Xylaria.

No mesmo período em que foram realizadas as entrevistas (estações seca e chuvosa), foram coletados macrofungos por busca ativa nos fragmentos de floresta da RBPT, explorando microhabitats propícios ao desenvolvimento dos ascomicetos e basidiomicetos (Mueller et al. 2004). A análise e identificação das amostras foram realizadas por especialistas do Departamento de Micologia da UFPE que integram o projeto CNPQ/ ICMBio 421241/2017-9, no qual se insere a presente pesquisa. Exsicatas representativas das espécies foram depositadas no Herbário URM, pertencente ao Departamento de Micologia da UFPE.

Figura 3. A) Álbum de fotografias dos macrofungos da Reserva Biológica de Pedra Talhada - RBPT utilizado como recurso visual. B) Amostras de macrofungos coletados na RBPT, empregadas como estímulo visual aos entrevistados. C) Entrevista realizada com moradora da comunidade do Arraial, Quebrangulo, Alagoas, Brasil.
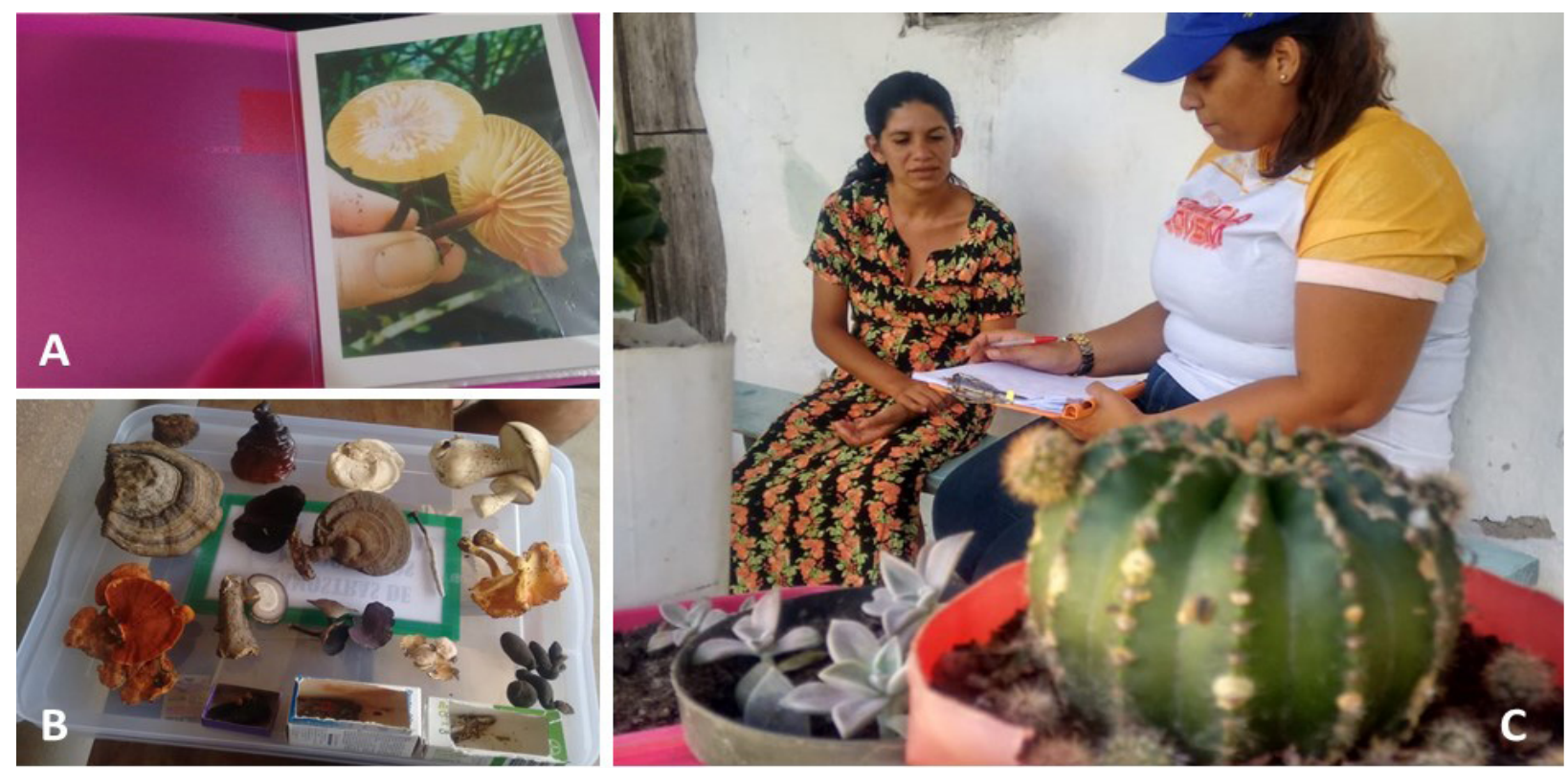

\section{Resultados E Discussão}

\section{Caracterização socioeconômica dos entrevistados}

No levantamento de informações sobre a percepção dos moradores que habitam o entorno da REBIO de Pedra Talhada sobre os macrofungos que ocorrem no local, foram entrevistadas 61 pessoas (jovens, 33\%; adultos, 41\%; idosos, 26\%), 59\% do gênero masculino e 41\% do gênero feminino. Entre os jovens, sem distinção de gênero, $85 \%$ concluiu o ensino fundamental e se encontram com o ensino médio concluído ou incompleto e dois iniciaram o ensino superior (Tabela 1). Entre os adultos, homens e mulheres, a maioria possui ensino fundamental incompleto (48\%), alguns concluíram o fundamental (28\%) e apenas $16 \%$ concluíram o ensino médio. Os idosos concluíram apenas o ensino fundamental, sendo considerados semialfabetizados (Tabela 1). 
Tabela 1. Nível de instrução nas diferentes faixas etárias dos entrevistados, moradores das comunidades do entorno da REBIO de Pedra Talhada, município de Quebrangulo, Alagoas, Brasil. Gênero: masculino (M), feminino $(F)$.

\begin{tabular}{|c|c|c|c|c|c|c|c|c|c|}
\hline \multirow{3}{*}{ Condição } & \multicolumn{9}{|c|}{ Faixa etária / gênero } \\
\hline & \multicolumn{3}{|c|}{$\begin{array}{c}\text { Jovens } \\
\text { (18 }-24 \text { anos) }\end{array}$} & \multicolumn{3}{|c|}{$\begin{array}{c}\text { Adultos } \\
(25-59 \text { anos })\end{array}$} & \multicolumn{3}{|c|}{$\begin{array}{c}\text { Idosos } \\
(\geq 60 \text { anos })\end{array}$} \\
\hline & $\mathbf{M}$ & F & Total (\%) & $\mathbf{M}$ & $\mathbf{F}$ & Total (\%) & $\mathbf{M}$ & F & Total $(\%)$ \\
\hline \multicolumn{10}{|c|}{ Sem instrução } \\
\hline & 0 & 0 & 0 & 0 & 0 & 0 & 5 & 2 & 44 \\
\hline \multicolumn{10}{|c|}{ Ensino Fundamental } \\
\hline Incompleto & 3 & 0 & 15 & 8 & 4 & 48 & 5 & 3 & 50 \\
\hline Completo & 1 & 2 & 15 & 2 & 5 & 28 & 1 & 0 & 6 \\
\hline \multicolumn{10}{|c|}{ Ensino Médio } \\
\hline Incompleto & 3 & 0 & 15 & 1 & 1 & 8 & 0 & 0 & 0 \\
\hline Completo & 6 & 3 & 45 & 0 & 3 & 12 & 0 & 0 & 0 \\
\hline \multicolumn{10}{|c|}{ Ensino Superior } \\
\hline Incompleto & 1 & 1 & 10 & 0 & 0 & 0 & 0 & 0 & 0 \\
\hline Completo & 0 & 0 & 0 & 0 & 1 & 4 & 0 & 0 & 0 \\
\hline TOTAL & 14 & 6 & 100 & 11 & 14 & 100 & 11 & 5 & 100 \\
\hline
\end{tabular}

O tempo de moradia dos entrevistados nas comunidades rurais do entorno da RBPT varia desde menos de cinco anos ( 2 pessoas) até mais de 50 anos (13 pessoas), porém 31\% mora há pelo menos 30 anos, o que favorece a percepção acerca dos macrofungos. Considerando a data em que a reserva biológica foi implantada, até o início do ano de 1989 parte desses moradores tinha suas residências e sítios dentro da mata e o tráfego de pessoas era livre, com fácil acesso aos recursos naturais, incluindo os macrofungos.

A agricultura de subsistência é praticada por 70\% dos moradores que habitam o entorno da RBPT, correspondendo à ocupação principal deles, 54\% com renda mensal de até um salário mínimo. Os não agricultores (30\%) exercem atividades diversas, atuando como professor ( 1 pessoa), merendeira (1 pessoa), comerciante (1 pessoa), domésticas (12 pessoas) e ajudante de serviços gerais (3 pessoas).

\section{Reconbecimento dos fungos}

Quando questionados se conheciam os macrofungos, em um primeiro momento $38 \%$ alegaram não saber o que eram fungos. Após serem apresentados a esses entrevistados um álbum de fotografias de espécies que ocorrem na RBPT e uma caixa contendo amostras recém-coletadas de macrofungos, apenas 8\% continuaram afirmando não os conhecer. Os relatos dos entrevistados evidenciam que, embora não façam uso, percebem a presença de espécies de Agaricaceae, Auriculariaceae, Pleurotaceae e Polyporaceae no ambiente, seja próximo às suas residências, seja na área florestada da RBPT. Nas listas de espécies apresentadas por Boa (2004), para diversos continentes e Gamboa-Trujillo et al. (2019), para o Equador, dentre as famílias e gêneros citados, também se destacam as Agaricaceae (Agaricus), Auriculariaceae (Auricularia), Pleurotaceae (Pleurotus), Polyporaceae (Lentinus), além de Suillaceae (Suillus) e Pluteaceae (Volvariela).

Quando induzidos a responder sobre como nomeavam os diversos tipos de macrofungos baseados nos estímulos visuais, foram citados 50 nomes (Figura 4), sendo mais comuns cogumelo (55\%), fungo (37\%), frieira (25\%), orelha de pau (23\%), mofo (13\%), doença de plantas (12\%). Alguns nomes foram citados apenas uma vez, 
como baronesa, cebola da mata, chapéu, chapéu de couro, guarda chuva, orelha de velho e ovinho. Usam palavras específicas, como frieira, para os cogumelos que se desenvolvem em fezes de herbívoros e que tendem a evitar, pois consideram que causam doenças. Ao se referirem às frieiras, os entrevistados recordavam de recomendações dos familiares mais velhos, com frases do tipo "Pisar na frieira dá coceira nos dedos" ou " Cuidado para não pisar na merda para não pegar frieira" (Quadro 1).

Quadro 1. Nomes atribuídos a fungos pelos informantes que habitam o entorno da REBIO de Pedra Talhada, Alagoas, Brasil.

\begin{tabular}{|c|c|}
\hline Percepção & Nomes atribuídos ao fungo \\
\hline Negativa & $\begin{array}{l}\text { Bicho que dá em lavoura; bolor; cogumelo no cocô de cavalo; cogumelo do mal; doença } \\
\text { em plantas; doença na laranja; espuma branca; frieira; fungo de alimentos; fungo de } \\
\text { frutas; fungo da rama; fungo de laranjeira; fungo de lavoura; fungo de plantações; fungo } \\
\text { de unha; fungos na couve; madeira podre; mofo; mofo branco; mofo na batata; mofo } \\
\text { na folha; mofo na laranja; mofo nas plantas; mofo no feijão de corda; mofo no inhame; } \\
\text { pano branco; praga que dá na plantação. }\end{array}$ \\
\hline Positiva & Cebola da mata; cogumelo do bem; cogumelo do sol; ovinho; decompositores. \\
\hline Neutra & $\begin{array}{l}\text { Baronesa; chapéu de couro; cogumelo que nasce nas árvores; cogumelo que nasce na } \\
\text { terra; fungo de cercado; fungo de chão; fungo da terra ; fungo de madeira; fungo de } \\
\text { cascas de árvores; fungo de pau; guarda chuva; orelha de burro; orelha de pau; orelha de } \\
\text { pau em Jaqueira; orelha de velho; orelha de velho nos paus; orelha de pau do cercado. }\end{array}$ \\
\hline
\end{tabular}

Espécies com esporocarpos pequenos, não incluídas como macrofungos, como Alternaria citri (Penz.) Mussat, também são percebidas pelos moradores das comunidades do entorno da RBPT, principalmente as fitopatógenas, por causarem prejuízos diretos nas plantações. Esses fungos foram referidos como bicho de lavoura, bolor, decompositores e espuma branca (Quadro 1).

Nas comunidades estudadas, os nomes populares atribuídos aos macrofungos frequentemente são complementados com termos referentes ao ambiente onde são encontrados, tais como orelha de pau, cogumelo do cocô, cogumelo do sol, cebola da mata, enquanto outros refletem a conotação positiva ou negativa atribuída à espécie, como cogumelo do bem, cogumelo do mal, frieira (Figura 4; Quadros 1-2). Observou-se que um mesmo nome, como orelha de pau, pode ser atribuído a mais de uma espécie, de um gênero ou de diferentes gêneros. Assim, nas entrevistas não era possível saber a qual delas a pessoa estava se referindo, a não ser quando a espécie estava incluída na caixa de amostras de macrofungos recém coletados ou nas fotografias de componentes da micobiota da RBPT.

Figura 4. Nomes atribuídos aos fungos pelos entrevistados das comunidades do entorno da REBIO de Pedra Talhada, município de Quebrangulo, Alagoas, Brasil. O tamanho da fonte representa a frequência com que a palavra foi citada.

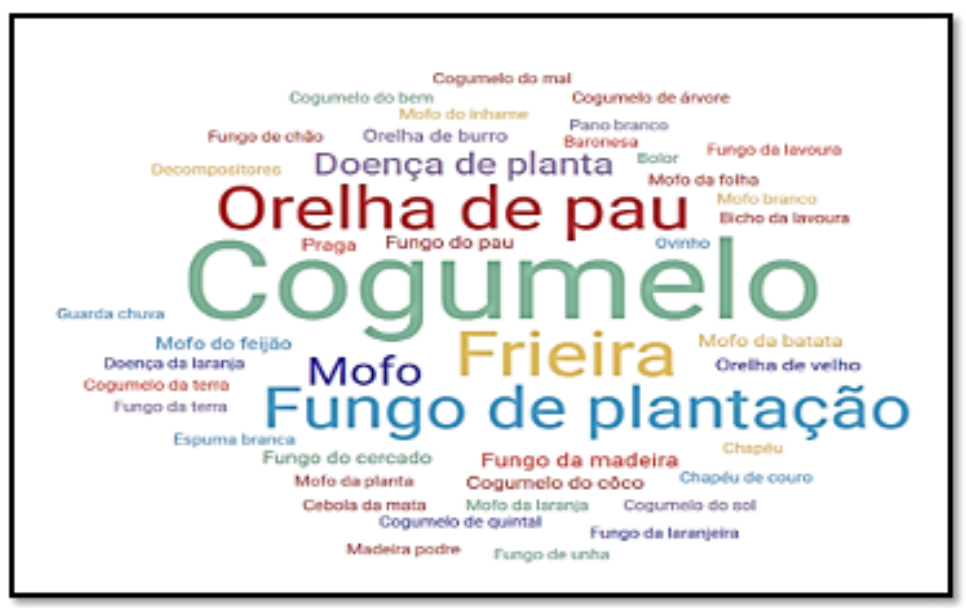


Nos relatos sobre nomes atribuídos por comunidades brasileiras aos fungos tem-se informação para grupos que habitam diferentes regiões do país, fornecidas em publicações de pesquisas etnomicológicas ou de outra natureza. Rodrigues (1905), por exemplo, em sua obra clássica sobre nomenclatura botânica de indígenas brasileiros, refere o termo urupês, empregado por índios Tupi-Guarani para diferentes espécies de basidiomicetos. Pesquisas etnomicológicas desenvolvidas em comunidades rurais do Nordeste e quilombolas do Centro-Oeste do país, evidenciam que o termo orelha de pau é aplicado para macrofungos de diferentes gêneros e espécies, como Ganoderma applanatum (Pers.) Pat., G. lucidum (Curtis) P. Karst. e Pycnoporus sanguineus (L.) Murrill (Souza et al. 2015; Cunha 2019).

Ao serem questionados a dizer em qual local encontram a maior presença de fungos, 50\% disseram em troncos mortos e caídos e nos cercados de madeira, 24\% em árvores, 13\% em fezes de herbívoros e 8\% no solo (Quadro 2). Eles conseguem perceber com mais frequência aqueles que estão crescendo sobre troncos mortos e caídos e em troncos de árvores vivas, mas também dão atenção aos que estão presentes nas fezes dos herbívoros, na serapilheira e no solo.

Quadro 2. Percepção dos informantes que habitam o entorno da REBIO de Pedra Talhada, Quebrangulo, Alagoas, em relação ao local de aparecimento dos fungos no ambiente. " $H$ " homem, "M" mulher, "J" jovem (1824), "A" adulto (25-59) e "I" idoso ( $\geq 60$ anos).

\begin{tabular}{|c|c|c|}
\hline $\begin{array}{l}\text { Local de } \\
\text { aparecimento }\end{array}$ & $\begin{array}{l}\text { Gênero/faixa } \\
\text { etária }\end{array}$ & Trechos de relatos \\
\hline \multirow{10}{*}{$\begin{array}{l}\text { Tronco morto } \\
\text { e caído }\end{array}$} & HI & "Os fungos nascem do pau velho, mas, não prejudica ninguém”. \\
\hline & MA & "Quando chove o tronco morto fica mais pesado e cresce..." \\
\hline & HI & "Não causam nenbum prejuízo porque não crescem no toco verde, só no tronco podre". \\
\hline & HI & "Quando chove o pau fica fraco e a madeira fica ruim eles aparecem". \\
\hline & HA & "Já viu cogumelos e orelhas de pau em madeira, mas não sabe como se chamam..." \\
\hline & HI & $\begin{array}{l}\text { "Nos paus podres de mulungu, os fungos crescem mais robustos, enquanto que nas árvores vivas } \\
\text { são menores". }\end{array}$ \\
\hline & MI & "...Percebia que nascia na madeira, mas não sabia o que era". \\
\hline & MA & "Cresce sempre em pau morto e podre... cresce mais nos cercados". \\
\hline & HI & "Madeira nova não dá muito fungo...". \\
\hline & HJ & $\begin{array}{l}\text { "Devido à seca que deu, tem muita madeira podre, com isso os fungos crescem mais, pois gostam } \\
\text { de madeira seca". }\end{array}$ \\
\hline \multirow{5}{*}{ Árvores } & MA & "Os fungos sugam as vitaminas das plantas e árvores". \\
\hline & MJ & "...Se alimenta de árvores molhadas". \\
\hline & MA & "Alguns fungos crescem em plantas vivas". \\
\hline & HI & "Nas plantaçôes há muitos prejuízos com fungos. Considera ruim, mal e venenosos". \\
\hline & HJ & "Dá em plantações de laranja e goiabeira". \\
\hline \multirow{2}{*}{$\begin{array}{l}\text { Fezes de } \\
\text { herbívoros }\end{array}$} & HI & "A frieira é um germe transmitido pelo cocô do cavalo, entre os dedos dos pés". \\
\hline & HA & "Os pais diziam cuidado para não pisar na merda para não pegar frieira”. \\
\hline \multirow{2}{*}{ Solo } & MJ & "Cogumelo nasce principalmente em terra molhada". \\
\hline & MA & "Cogumelos que saem da terra chama-se de frieira". \\
\hline
\end{tabular}

Foi frequente cada entrevistado associar o macrofungo com o ambiente onde é encontrado, distinguindo espécies terrestres de Chlorophyllum, Macrolepiota, Cantharellus e Tricholoma, como fungo de solo, fungo de chão, fungo da terra, daquelas que ocorrem em troncos de árvores vivas, como as dos gêneros Auricularia, Mycena, Favolus e Pleurotus, ou em troncos mortos, como as representantes dos gêneros Ganoderma, Fomes, Schizophyllum e Pycnoporus. 
Tal comportamento não está restrito à cultura nordestina ou às características ecológicas do ambiente da RBPT, sendo também referido para comunidades sul-americanas (Ruan-Soto et al. 2004; Cardoso et al. 2010), africanas (Teke et al. 2018) e asiáticas (Borah et al. 2018).

As comunidades que habitam o entorno da RBPT percebem a relação dos macrofungos com a variação dos fatores climáticos sobre a esporulação. Uma das idosas entrevistadas, por exemplo, relatou que uma espécie de Marasmius (Figura 5 - A), que conhece como fungo vermelho ou estrondo, aparece em quintais ou na mata logo após fortes trovoadas.

Figura 5. Principais espécies de macrofungos indicadas pelos informantes que habitam o entorno da REBIO de Pedra Talhada, Alagoas. A) Marasmius sp.; B) Cookeina tricholoma; C) Fomes fasciatus; D) Ganoderma australe; E) Geastrum sp.

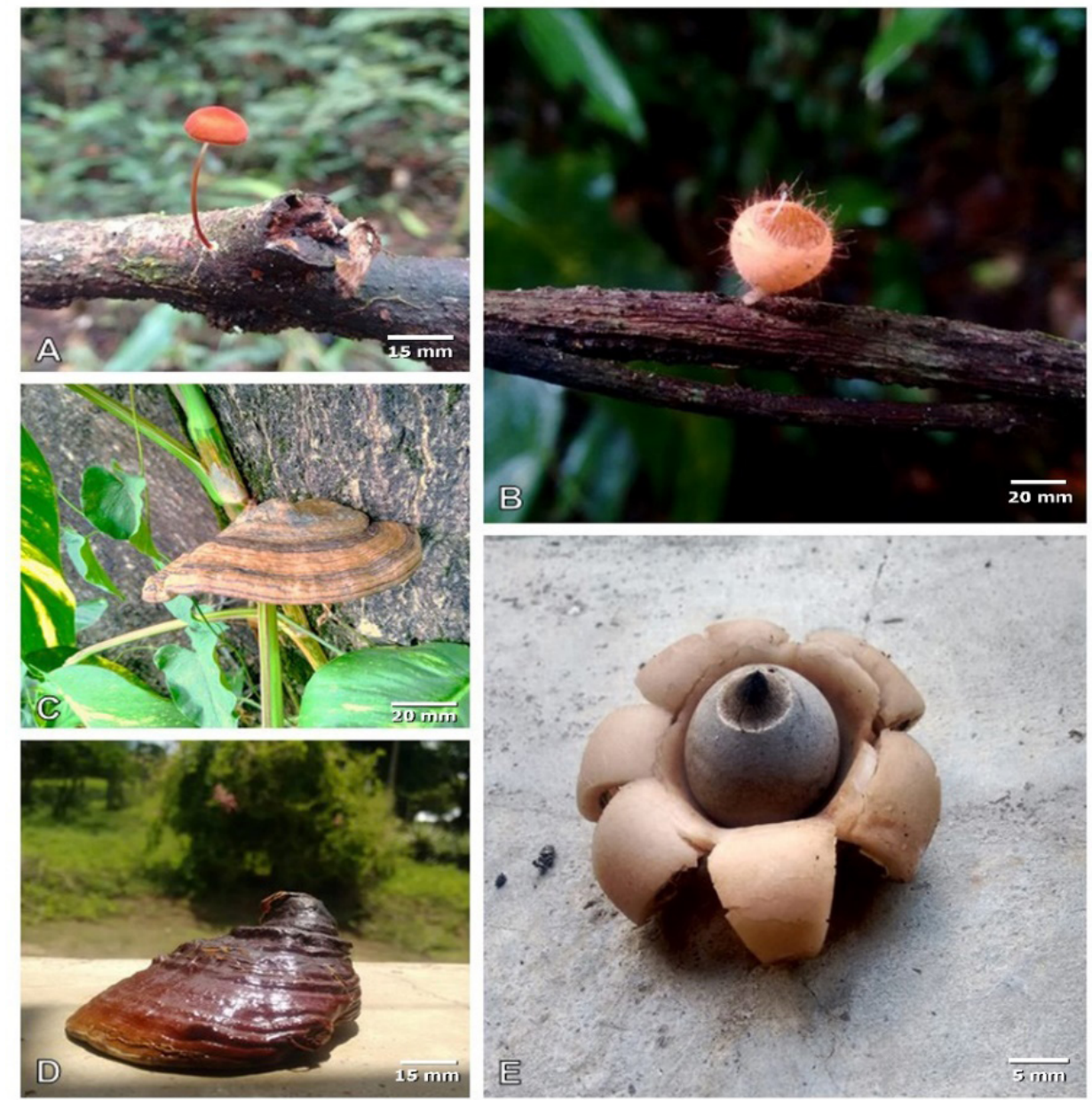

Ao serem questionados sobre qual período do ano que os macrofungos são vistos com maior frequência, 79\% relacionaram a esporulação com o período chuvoso, $18 \%$ mencionaram o período de seca e 3\% não souberam responder. Alguns entrevistados comentaram que, apesar de haver maior incidência no período chuvoso, o aparecimento desses organismos depende do substrato que o fungo se desenvolve, de modo que se houver umidade e condições favoráveis não depende exclusivamente da chuva. Mesmo citando o período chuvoso como mais favorável, muitos abriram parênteses para dizer que surgiam em madeira molhada, na primavera, em locais verdes e úmidos ou em qualquer época do ano. A percepção dos entrevistados sobre o comportamento dos macrofungos em relação aos fatores climáticos é semelhante à observada por Sousa et al. (2015) em uma comunidade rural do semiárido nordestino e por Cunha (2019), em uma comunidade quilombola que habita o cerrado no estado de Goiás, Centro-Oeste do país. 
Grupos humanos que vivem em regiões tropicais, onde as espécies decompositoras de madeira são mais abundantes, se relacionam menos com fungos que os habitantes de regiões temperadas, onde predominam espécies terrestres, cujos corpos frutíferos são mais palatáveis (Guzmán 1983). Segundo Mapes et al. (2002), ao migrarem de regiões temperadas para os trópicos, as culturas ameríndias não encontraram as espécies de fungos que já conheciam como úteis e passaram a utilizar mais o recurso vegetal que o micológico. No inventário dos macrofungos presentes na RBPT, realizado entre 2018 e 2019, foram obtidos 5000 espécimes de fungos, representando pouco mais de 800 espécimes de Basidiomycota e cerca de 400 espécimes de Ascomycota. O inventário evidenciou a ocorrência de 12 famílias de basidiomicetos, destacando-se pelo número de gêneros Polyporaceae e Hymenochaetaceae e 12 famílias de ascomicetos, destacando-se Xylariaceae (Figura 6 A-B).

Figura 6. Número de ocorrências de gêneros por famílias de macrofungos registradas na REBIO de Pedra Talhada, Quebrangulo, Alagoas. A) Basidiomycetes. B) Ascomycetes.

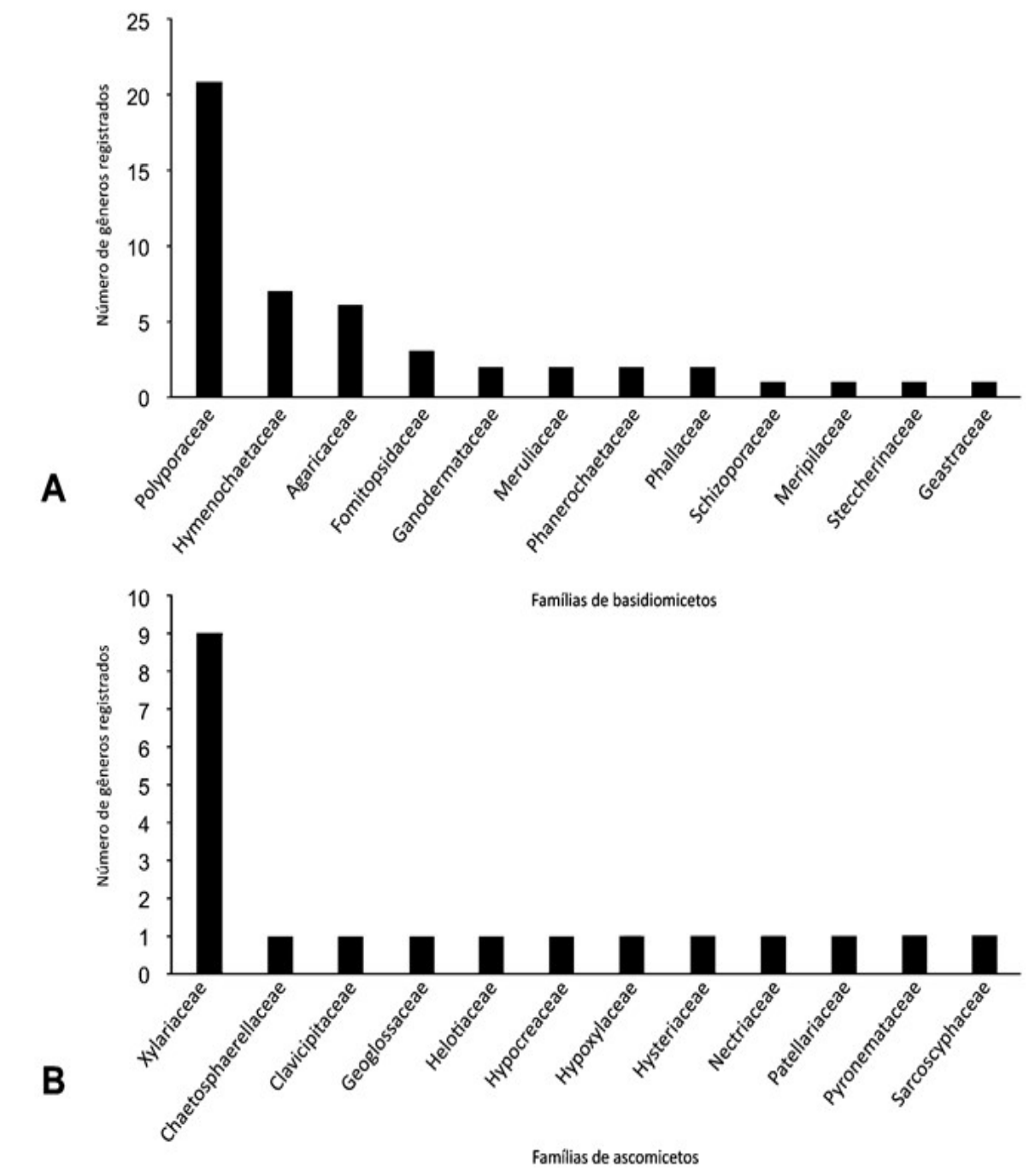

Xylariaceae é a família com maior número de gêneros e espécies dentre os ascomicetos presentes na RBPT (Figura 6 - B), representada por Annulobypoxylon (1 sp), Biscognianxia (3 spp), Camillea (1 sp), Daldinia (1 sp), Kretzschmaria (1 sp), Rhopalostroma (1 sp), Rosellinia (1 sp), Stilbohypoxylon (2 spp) e Xylaria (16 spp). Na literatura mundial, espécies de Xylariaceae, principalmente do gênero Xylaria, são relatadas como medicinais, ritualísticas, lúdicas, mitológicas, zoocomestíveis e ornamentais, como por exemplo nas etnias Kichwa e Chachis do Equador 
(Gamboa-Trujillo et al. 2019). Embora Xylaria seja um gênero bem representado na Floresta Atlântica, constatouse que a maioria das espécies não é percebida pelos moradores do entorno da RBPT.

Basidiomicetos das famílias Polyporaceae, Ganodermataceae e Fomitopsidaceae estão representados na RBPT por 56 espécies, pertencentes aos gêneros Amauroderma, Favolus, Flabellophora, Fomes, Fomitopsis, Ganoderma, Polyporus, Pycnoporus, Rhodofomitopsis, Rigidoporus, Trametes, entre outros (Figura 6 - A). Dentre as espécies que compõem a micobiota da RBPT inclui-se Pycnoporus sanguineus (L.) Murrill, relatada como comestível, medicinal e ornamental em comunidades indígenas da etnia Hoti, na Venezuela e Kichwas amazônicos e Secoyas, no Equador (Zent et al. 2004; Zent 2008; Gamboa-Trujillo et al. 2019). Para a região Norte do Brasil, Fidalgo (1965) e Fidalgo e Hirata (1979) referem o uso de P. sanguineus por indígenas como-anti-hemorrágica e comestível. No Amapá, Sotão e Figueiredo (1996) referem seu uso para acabamento de peças de barro por quilombolas. Em Goiás, $P$. sanguineus é utilizada como medicamento para atraso no ciclo menstrual (Cunha 2019) e no Piauí é empregada por comunidades rurais da Caatinga no tratamento da asma, associada com ovos de gastrópode (Sousa 2020). Apesar da diversidade de categorias de uso de P. sanguineus em diferentes culturas e países, incluindo o Brasil, não foram obtidos relatos de sua utilização pelos moradores das comunidades estudadas.

Amauroderma sprucei (Pat.) Torrend, também presente na RBPT, tem distribuição de Norte ao Sul do Brasil, sendo encontrada nos domínios da Amazônia, da Caatinga e da Mata Atlântica (Flora do Brasil 2020). Na Venezuela, a espécie Amauroderma of. omphalodes (Berk.) Torrend é utilizada como comestível e medicinal (Zent et al. 2004; Zent 2008), porém não foram obtidos registros da utilização de espécies de Amauroderma pela população que vive no entorno da RBPT. Quando se apresentou aos entrevistados um exemplar de $A$. sprucei recém-colhido na floresta, 12 deles (20\%) relataram que observaram o macrofungo no ambiente, porém não lhe atribuíram nome e não referiram utilidade para a espécie.

No Brasil, tem-se relatos de ocorrência de espécies do gênero Ganoderma desde o Norte até o Sul do país (Flora do Brasil 2020). Para o Nordeste brasileiro Sousa et al. (2017) relatam uso de Ganoderma applanatum (Pers.) Pat., na composição de um xarope preparado com a casca de uma planta leguminosa conhecida como angico, para tratamento da gripe; relatam também uso da espécie para doenças dos sistemas digestivo e reprodutor. No Equador, Ganoderma australe (Fr.) Pat. é utilizada no tratamento de enfermidades respiratórias por comunidades indígenas da etnia Kichwas amazônicos (Gamboa-Trujillo et al. 2019). Embora não se tenha encontrado muitas evidências de uso medicinal houve relatos de chás preparados com orelhas de pau (basidiomicetos), por informantes de diferentes gêneros e faixas etárias. Um idoso, residente na comunidade Juliana, relatou uso de $G$. australe (Figura 5 - D) para tratamento de asma que sofria há 40 anos e informou ter sido curado da enfermidade com o uso contínuo do chá feito com a orelha de pau.

Basidiomicetos do gênero Fomes são utilizados há séculos para fins medicinais em países asiáticos e nos últimos anos, metabólitos presentes nos basidiomas foram estudados, principalmente na investigação de potencialidade para tratamento de câncer (Bal e Akgül 2018). Um comerciante adulto, residente da comunidade Boa Vista, citou o uso de Fomes fasciatus (Sw.) Cooke (Figura 5 - C) para o tratamento de câncer. Um jovem, residente na comunidade Juliana, e uma idosa, residente na comunidade Pedra Talhada, também relataram uso medicinal de orelhas de pau no tratamento de tosse e falta de ar, porém não foi possível a identificação das espécies usadas, não disponíveis no local no momento da entrevista nem nas amostras da RBPT e nas fotografias a eles apresentadas.

Em concordância com Guzmán (1983) e Mapes et al. (2002), apesar da riqueza de macrofungos carnosos os moradores das comunidades estudadas não os utilizam como recurso alimentício. Apenas o ascomiceto Cookeina tricholoma (Mont.) Kuntze (Figura 5 - B), referido como cebola da mata, foi citado como comestível por um idoso, que teve oportunidade de caçar livremente na floresta antes de ser considerada Unidade de Conservação. Esta espécie ocorre em fragmentos de Floresta Atlântica no Nordeste e Sul do Brasil (Flora do Brasil 2020), porém este é o seu primeiro relato como comestível por comunidades da Mata Atlântica e Nordeste do Brasil. Espécies desse gênero, como C. tricholoma, C. speciosa (Fr.) Dennis e C. sulcipes (Berk.) Kuntze estão presentes em listas de fungos 
comestíveis por povos latino-americanos e africanos, nas Guianas, Panamá, Costa Rica, México e República do Congo (Boa 2004; Henkel et al. 2004; Kamalebo et al. 2018). Em países asiáticos e mesoamericanos C. tricholoma tem registro como comestível por comunidades tradicionais da Malásia (Chang et al. 2004) e usos lúdico, medicinal e mitológico foram relatados para comunidades indígenas do Equador e do México (Ruan-Soto et al. 2009; Gamboa-Trujillo et al. 2019).

Fungos com fácil liberação de esporos são atraentes para as crianças na hora da brincadeira, pois formam uma nuvem de esporos ao serem tocados (Cunha 2019). O uso como brinquedo foi relatado por uma mulher jovem da comunidade de Pai Vicente para um basidiomiceto do gênero Geastrum (Figura 5 - E), localmente conhecido como ovinho. Outra jovem, residente na comunidade Juliana, relatou que brincava com espécies de basidiomicetos que chamava de frieira. O uso lúdico é raramente relatado para macrofungos, mas espécies de Agaricus e Coprinus foram relatadas como objeto lúdico em comunidades rurais do estado do Piauí, Nordeste do Brasil (Sousa et al. 2015). Em outras comunidades sul-americanas encontra- se relatos do uso de esporocarpos de Lycogala epidendrum (L.) Fr., (Myxomycota) e Cookeina tricholoma (Ascomycota) como brinquedos de crianças das etnias Kichwas e Epera, no Equador (Gamboa-Trujillo et al. 2019). Em comunidades latino-americanas, RuanSoto et al. (2009) relatam o uso de C. sulcipes e C. tricholoma como objeto lúdico no México.

\section{CONCLUSÕES}

Na REBIO de Pedra Talhada e áreas adjacentes ocorrem espécies de macrofungos (basidiomicetos e ascomicetos) com potencial de utilização como recursos alimentícios e medicinais. As comunidades que vivem no seu entorno percebem a presença dos fungos e a relação deles com fatores bióticos e abióticos, principalmente como bioindicadores de variações climáticas, mas não costumam utiliza-los no dia a dia, podendo ser enquadradas como não micófilas.

A pesquisa traz os primeiros relatos de utilização de espécies presentes em fragmentos de Floresta Atlântica por comunidades nordestinas: Cookeina tricholoma, comestível, Geastrum sp., lúdico, Ganoderma australe e Fomes fasciatus, medicinal.

\section{Agradecimentos}

Os autores agradecem aos moradores das comunidades de Pedra Talhada, Carangueja, Sítio do Gavião, Juliana, Arraial, Riachão de Cima, Riachão de Baixo, Pai Vicente e Boa Vista (Quebrangulo - Alagoas), que se disponibilizaram a trocar informações e à associação NORDESTA - Reflorestamento e Educação. Agradecem ainda ao Sistema de Biodiversidade e Conservação (SISBIO) do Instituto Chico Mendes (ICMBio), ao Comitê de Ética em Pesquisa (CEP) da UFPE e ao CNPq e FACEPE, pelo apoio financeiro.

\section{REFERÊNCIAS}

Albuquerque UP, Lucena, FRP, Lins Neto, EMF. 2008. Seleção e escolha dos participantes da pesquisa, pp 21-40 In: Albuquerque UP, Lucena RFP, Cunha LVFC. (Orgs.). Métodos e técnicas na pesquisa etnobotânica. 2ed. NUPEEA, Recife.

Apiamö RM, Autuori J, Ishikawa NK, Martins MS, Menolli Jr N, Sanuma C, Sanuma LR, Sanuma M, Sanuma OI, Tokimoto K (Eds). 2016. Enciclopédia dos alimentos Yanomami (Sanöma): Cogumelos. Instituto Socioambiental, São Paulo. 108 pp. 
Bal C, Akgül H. 2018. Pharmacological properties of Fomes fomentarius. In: International Eurasian Conference on Science, Engineering and Technology, Ankara, Turkey. pp. 1195-1197.

Boa ER. 2004. Wild edible fungi: A global overview of their use and importance to people. Food and Agriculture Organization of the United Nations, Rome.

Borah N, Semwal RL, Garkoti SC. 2018. Ethnomycological knowledge of three indigenous communities of Assam, India. Indian Journal of Traditional Knowledge, 17(2): 327-335.

Cardoso DBOS, Queiroz LP, Bandeira FP, Góes-Neto A. 2010. Correlations between indigenous Brazilian folk classifications of fungi and their systematics. Journal of Etnobiology, 30(2): 255-267.

Chang YS, Lee SS. 2004. Utilization of macrofungi species in Malaysia. Fungal Diversity, 15: 15 - 22.

Cunha EL. 2019. Etnomicologia: a produção científica global e o conhecimento etnomicológico em uma comunidade quilombola do Cerrado. Mestrado em Recursos Naturais do Cerrado, Universidade Estadual de Goiás.

Santana MDF, Costa ADL, Gomes ESC, Guimarães LES. 2019. Ocurrencia y apuntes etnomicológicos sobre Phallus indusiatus (Phallaceae, Basidiomycota) en la Reserva Extrativista Tapajós-Arapiuns, Pará, Brasil. Acta Botanica Mexicana 126: e1436. DOI: $10.21829 / \mathrm{abm} 126.2019 .1436$

Fidalgo O, Poroca DJM. 1986. Etnomicologia brasileira. Boletim de Micologia, 3(1): 9-19.

Fidalgo O, Hirata D. 1979. Etnomicologia Caiabi, Txicâo e Txucarramâe. Rickia, 8: 1-5.

Fidalgo O, Prance GT. 1976. The ethnomycology of the Sanama Indians. Mycologia, 68: 201-210.

Fidalgo O. 1965. Conhecimento micológico dos índios brasileiros. Rickia, 2:1-10.

Flora do Brasil 2020 (em construção). Jardim Botânico do Rio de Janeiro. Disponível em: <http://floradobrasil.jbrj.gov. br/>. Acesso em 17 de janeiro de 2020.

Gamboa-Trujillo P, Wartchow F, Cerón-Martinez C, Andi D, Uwinjin P, Grefa ME, Chimbo E, Chimbo J, Payaguaje J, Payaguaje N, Payaguaje D, Licuy V, Lópes V, Mendua M, Criollo M, Jati M, Cruz S, Calazacón M, Flores S, Aules E, Aigaje C, Aro M, Morales A, Murillo Y, Farinango G, Gilbertoni TB. 2019. Edible Mushrooms of Ecuador: consumption, myths and implications for conservation. Ethnobotany Research \& Applications, 18:38. http://dx.doi.org/10.32859/ era.18.38.1-15

Góes-Neto A, Bandeira FP. 2002. A review of the Ethnomycology of indigenous people in Brazil and its relevance to ethnomycological investigation in Latin America. Revista Mexicana de Micologia, 17:11-16.

Guzmán, G. 1983. Los hongos de la península de Yucatán II. Nuevas exploraciones y adiciones micológicas. Biotica, 8:71-100.

Henkel TW, Aime C, Chin C, Christopher A. 2004. Edible mushrooms from Guyana. Micologist, 18 (3): 104-111.

IBGE (Instituto Brasileiro de Geografia e Estatística). 2010. Aspectos físicos de São Raimundo Nonato. Disponível em: <http://www.ibge.gov.br/cidadesat/topwindow.htm?1>. Acesso em: 10 jul. 2018. 
Ishikawa NK, Vargas-Isla R, Chaves RS, Cabral TS. 2012. Macrofungos da Amazônia: importância e potencialidades. Ciência e Ambiente, 44: 129-139.

Kamalebo HN, Malale HNSW, Ndabaga CN, Degreef J, Kesel A. 2018. Uses and importance of wild fungi: traditional knowledge from the Tshopo province in the Democratic Republic of the Congo. Journal of Ethnobiology and Ethnomedicine 14:13. DOI 10.1186/s13002-017-0203-6

Kotowski MA. 2019. History of mushroom consumption and its impact on traditional view on mycobiota - an example from Poland. Microbial Biosystems, 4(3): 1-13.

Londoño- Castañeda PA, Buril MLL, Rego-Cunha IP, Silva NH, Honda NK, Pereira EC, Andrade LHC. 2017. Lichens used in the traditional medicine by the Pankararu Indigenous Community, Pernambuco-Brazil. Global Journal of Science Frontier Research: C Biological Science, 17(4): 15-22.

Mapes C, Bandeira FPSF, Caballero J, Góes-Neto A. 2002. Mycophobic or mycophilic? A comparative ethnobiological study between Amazonia and Mesoamerica, pp180-188 In: Stepp JR, Wyndham FS, Zarger RK. (Org.). Ethnobiology and Biocultural Diversity: Proceedings of the Seventh International Congress of Ethnobiology. Athens: The University of Georgia Press, 2002.

Morales B, Cáceres R, Flores R. 2003. Contribución al conocimiento de los hongos comestibles de Guatemala. Revista Científica, 1 (1): 2-24.

Mueller GM, Bills GF, Foster MS. 2004. Biodiversity of Fungi: Inventory and Monitoring Methods. Elsevier Academic Press, Burlington, MA, 777 pp.

Nusbaumer L, Barbosa MRV, Thomas WW, Alves MV, Loizeau P-A, Spichiger R. 2015. Flora e vegetação da Reserva Biológica de Pedra Talhada. In: Studer A, Nusbaumer L, Spichiger R. (Eds.). Biodiversidade da Reserva Biológica de Pedra Talhada (Alagoas, Pernambuco - Brasil). Boissiera. 68: 59-121.

Prance G. 1973. The mycological diet of the Yanomami Indians. Mycologia, 65: 248-250.

Rodrigues, J B. 1905. Mbaé kaá tapyiyetá enoyndaua ou a botanica e a nomenclatura indigena. Memoria apresentada ao Terceiro Congresso Cientifico Latino Americano. Rio de Janeiro. vi, 87 pp.

Ruan-Soto F, Cifuentes J, Marianca MR, Limon F, Pérez-Ramírez L, Sierra S. 2009. Uso y manejo de hongos silvestres en dos comunidades de la Selva Lacandona, Chiapas, México. Revista Mexicana de Micología, 29: 61-72.

Ruan-Soto F. 2017. 50 años de Etnomicología en México. Lacandonia, 1(1):97-108.

Ruan-Soto F, Garibay-Orijel R, Cifuentes J. 2004. Conocimiento micológico tradicional en la planicie costera del Golfo de México. Revista Mexicana de Micología, 19: 57-70.

Sotão HM, Figueiredo T. 1996. Utilização do fungo Pycnoporus sanguineus (L.: Fr.) Murr. na cerâmica do Maruanum, Amapá. Boletim do Museu Paraense Emilio Goeldi, ser. Bot., 12(1): 15-20.

Sousa SB. 2020. Fungos e plantas com potencial fungicida conhecidos ou utilizados por comunidades rurais no nordeste do Brasil. Doutorado em Desenvolvimento e Meio Ambiente, PRODEMA, Universidade Federal do Piauí. 
Sousa SB, Lucena RFP, Barros RFM, Rocha JRS. 2015. Classificação folk dos macrofungos por uma comunidade rural no semiárido do Nordeste do Brasil. Espacios, 36 (21): 18.

Sousa SB, Rocha JRS, Lucena RFP, Barros RFM. 2017a. Percepção sobre os macrofungos em uma comunidade rural na Caatinga, Nordeste do Brasil. Gaya Scientia, 11 (2): 231-241. http://dx.doi.org/10.21707/gs.v11.n02a016

Sousa SB, Rocha JRS, Lucena RFP, Barros RFM. 2017b. Uso de macrofungos em região de Caatinga no Nordeste do Brasil. Gaya Scientia, 11 (3): 101-113. http://dx.doi.org/10.21707/gs.v11.n03a09

Teke NA, Kinge TR, Bechem E, Nji TM, Ndam LM, Mih AM. 2018. Ethnomycological study in the Kilum-Ijim mountain forest, Northwest Region, Cameroon. Journal of Ethnobiology and Ethnomedicine, 14:25. https://doi.org/10.1186/ s13002-018-0225-8

Trierveiler-Pereira L, Sulzbacher MA, Baltazar JM. 2018. Diversidade de fungos brasileiros e alimentação: o que podemos consumir? In: III Fórum Ambiental de Angatuba, 2018, Angatuba-SP. Resumo Expandido nos Anais do III Fórum Ambiental de Angatuba, 2018.

Trutmann P, Holgado ME, Quispe A, Luque A. 2012. Native mushrooms, local knowledge, and potential for food and health in the Peruvian Andes. Annual Report 2012, Global Mountain Action, 1: 33pp.

Tscharner T, Duda GP, Oliveira VP, Silva CMS, Nusbaumer L, Silva Filho AF. 2015. Parâmetros abióticos da Reserva Biológica de Pedra Talhada pp.39-57 In: Studer A, Nusbaumer L, Spichiger R. (Eds.). Biodiversidade da Reserva Biológica de Pedra Talhada (Alagoas, Pernambuco - Brasil). Boissiera. v. 68.

Vargas-Isla R, Ishikawa NK, Py-Daniel V. 2013. Contribuições etnomicológicas dos povos indígenas da Amazônia. Biota Amazônia, 3(1): 58-65.

Wasson RG. 1957. Seeking the Magic Mushroom. Life 42: 100-120.

Yanomami FCP, Vieira MARM, Ishikawa NK (orgs.). 2019. Marasmius yanomami: o fungo que as mulheres Yanomami usam na cestaria. Instituto Socioambiental, São Paulo. 72 pp.

Zent EL, Zent S, Iturriaga T. 2004. Knowledge and use of fungi by a mycophilic society of the Venezuelan Amazon. Economic Botany, 58(2): 214-226.

Zent EL. 2008. Mushrooms for Life among the Joti in the Venezuelan Guayana. Economic Botany, 62(3): 471-481.

Zuchiwschi E, Fantini AC, Alves AC, Peroni N. 2010. Limitações ao uso de espécies florestais nativas pode contribuir para a erosão do conhecimento ecológico tradicional e local de agricultores. Acta Botânica Brasilica, 24(1): 270-282. 